\title{
An Ontology-based Framework for XML Semantic Integration
}

\author{
Isabel F. Cruz Huiyong Xiao Feihong Hsu \\ Department of Computer Science \\ University of Illinois at Chicago \\ \{ifc, hxiao, fhsu\}@cs.uic.edu
}

\begin{abstract}
$X M L$ is becoming the standard for data interchange on the web. However, XML and its schema languages do not express semantics but rather structure, such as nesting information. Therefore, semantically equivalent documents often present different document structures. In this paper, we provide an ontology-based framework that aims to make two XML documents interoperate at the semantic level while retaining their nesting structure. In our global-asview approach, we generate an RDF ontology for each of the participating XML documents, which preserves the nesting structure of the document. An RDF global ontology is the result of merging the individual ontologies. The global ontology unifies the query access and establishes semantic connections among the underlying individual databases. We consider two types of queries: those that are posed on the global ontology and those that are posed on any of the $X M L$ documents, in a P2P fashion. The former type is processed using query translation from an RDF query to an $X M L$ query. The latter type entails bidirectional query processing: the translation from an XML query to an $R D F$ query followed by the translation from an RDF query to an XML query. To ensure the correctness of the answer to the query in the latter case, we introduce the concept of reversibility of the query translation.
\end{abstract}

\section{Introduction}

\subsection{Problem description}

One of the primary obstacles in Semantic Web applications is the heterogeneity of the distributed data sources. These heterogeneities can be classified as syntactic, schematic, and semantic heterogeneities [3]. Our previous work gives an example of solving schematic heterogeneities, that is, those that arise from using different schemas to represent the same data [10]. In this paper, we focus on the problem of semantic interoperability between different XML sources. In doing so, we propose an approach for integration of heterogeneous XML sources and query processing across these XML sources.

XML documents that conform to different schemas may represent data with similar semantics. Therefore, a user must construct queries in accordance to an XML document's structure to retrieve fragments of information that have the same meaning. This fact makes the formulation of queries on heterogeneous XML sources a nontrivial burden to the user. Furthermore, this shortcoming of XML impedes the interoperability of XML sources since the reformulation of XML queries has to eliminate the structural differences of the queries while presenting the same semantics. Let us illustrate the problem using a running example as shown in Figure 1.

Example 1.1 Figure 1 gives two XML schemas $\left(S_{1}\right.$ and $\left.S_{2}\right)$ with their documents $\left(D_{1}\right.$ and $\left.D_{2}\right)$, which are represented as trees. It is obvious that $S_{1}$ and $S_{2}$ both represent a many-to-many relationship between two concepts: book and author (equivalently denoted by article and writer in $S_{2}$ ). However, structurally speaking, they are different: $S_{1}$ (book-centric schema) has the aut hor element nested under the book element, whereas $S_{2}$ (author-centric schema) has the article element nested under the writer element. Suppose our query target is "Find all the authors of the publication $b_{2}$." The XML path expressions that are used to define the search patterns in the two schema trees can be respectively written as /books/book [ [booktit le="b2"] /author/ename and/writers/writer [article / [etitle="b2"]/@ful Iname, where the contents in the square brackets specify the constraints for the search patterns. We notice that although the above two search patterns refer to semantically equivalent concepts, they follow two distinct XML paths.

\subsection{Semantic integration of XML documents}

The diversity of the XML queries (referred to in this paper as structural queries) results from the diversity of pos- 


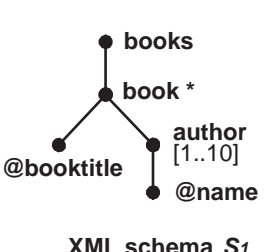

XML schema $S_{1}$

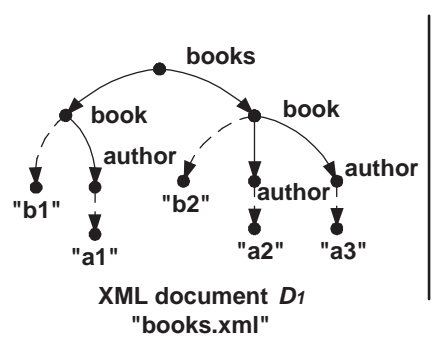

"books.xml"

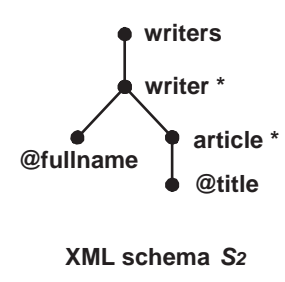

XML schema $\mathbf{S}$

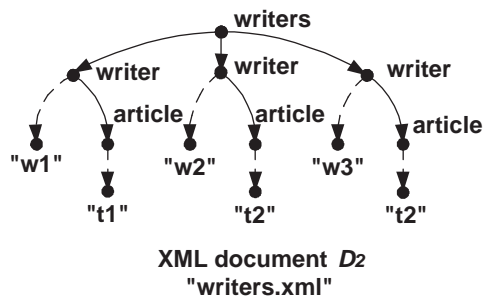

Figure 1. An example of structural heterogeneity between XML documents.

sible XML schemas (also called structural schemas) for a single conceptual model. In comparison, the schema languages that operate on the conceptual level (called conceptual schemas) are structurally flat so that the user can formulate a determined query (called conceptual query) without considering the structure of the source. RDF Schema (RDFS) [15], DAML+OIL, and OWL are examples of languages used to create conceptual schemas. There are currently many attempts to use conceptual schemas $[1,2,10]$ or conceptual queries $[8,9]$ to overcome the problem of structural heterogeneities among XML sources.

In this paper, we propose an approach to integrate XML sources and handle queries in the integrated system by using a bidirectional query translation algorithm. We choose to use the global-as-view (GaV) approach [7] for the integration of XML sources. For the sake of simplicity, we use RDFS to model the ontologies (instead of DAML+OIL or OWL). This choice limits the expressiveness of the model, so that some axioms (e.g., disjunction of two concepts) cannot be used.

In particular, we first transform the heterogeneous XML sources into local RDF-based ontologies (defined using the RDFS space [6]), which are then merged into the RDF global ontology. This transformation process encodes the mapping information between each concept in the local $\mathrm{RDF}$ ontology and the path to the corresponding element in the XML source. The ontology merging process is semiautomatically performed by utilizing the PROMPT algorithm [16]. Apart from the global ontology, the merging process also produces a mapping table, which contains the mapping information between concepts in the global ontology and concepts in the local RDF ontologies. In our approach, we can translate a query posed against the global ontology into sub-queries over the sources. We can also translate a query posed against an XML source to an equivalent query against any other XML source. Given that we choose a $\mathrm{GaV}$ approach, the global ontology is a view over the local ontologies, therefore the process of mapping a query over the global ontology to queries over the local ontologies is straightforward.

\subsection{Contributions}

In brief, we make the following contributions in this paper:

- We propose an approach for using an ontologybased mediation architecture to integrate heterogeneous XML sources. The mediation integrates both the XML nesting structure and the domain structure expressed by RDFS to enable semantic interoperation between the XML sources by hiding their structural heterogeneities. This integration process is lossless with respect to the nesting structure of the XML document.

- We extend RDFS by defining additional metadata. These metadata encode the nested structure of the XML Schema in the RDF schema.

- Finally, we describe an algorithm for translating the query back and forth between XQuery and RDQL. The translation is based on a mapping table and the principle of preserving the nesting structure on the XML sources.

The paper is organized as follows. Section 2 describes related work. Section 3 describes the architecture of our approach. Data integration and query processing, which are the two key points in our approach, are discussed respectively in Sections 4 and 5. We draw conclusions and discuss future work in Section 6.

\section{Related Work}

There are a number of approaches addressing the problem of data integration or interoperability among XML sources. The approaches proposed can be classified into the following three categories.

\subsection{Semantic integration}

High-level Mediator Amann et al. propose an ontologybased approach to the integration of heterogeneous 
XML Web resources in the C-Web project $[1,2]$. The proposed approach is very similar to our approach except for the following differences. The first difference is that they use the local-as-view (LaV) approach [7] with a hypothetical global ontology that may be incomplete. The second difference is that they do not retain the XML documents' structures in their conceptual mediator so they cannot deal with the reverse query translation (from the XML sources to the mediator). Our previous work [10] involved a layered approach for the interoperability of heterogeneous web sources, but the nesting structure associated with XML was lost in the mapping from XML data to RDF data.

Direct Translation Klein proposes a procedure to transform XML data directly into RDF data by annotating the XML documents via external RDFS specifications [13]. The procedure makes the data in XML documents available for the Semantic Web. However, since the proposed approach does not consider the document structure of XML sources, it can not propagate queries from one XML source to another XML source.

Encoding Semantics The Yin/Yang Web [17] proposed by Patel-Schneider and Siméon address the problem of incorporating the XML and RDF paradigms. They develop an integrated model for XML and RDF by integrating the semantics and inferencing rules of RDF into XML, so that XML querying can benefit from their RDF reasoner. But the Yin/Yang Web does not solve the essential problem of query answering across heterogeneous sources, that is, sources with different syntax or data models. It also cannot process higher-level queries such as RDQL. Lakshmanan and Sadri also propose an infrastructure for interoperating over XML data sources by semantically marking up the information contents of data sources using application-specific common vocabularies [14]. However, the proposed approach relies on the availability of an application-specific standard ontology that serves as the global schema. This global schema contains information necessary for interoperability, such as key and cardinality information for predicates. This approach has the same problem as the Yin/Yang Web, that is, higher-level queries can not be processed downwards to XML queries.

\subsection{Query languages}

CXQuery [9] is a new XML query language proposed by Chen and Revesz, which borrows features from both SQL and other XML query languages. It overcomes the limitations of the XQuery language by allowing the user to define views, explicitly specify the schema of the query an- swers, and query through multiple XML documents. However, CXQuery does not solve the issue of structural heterogeneities among XML sources. The user has to be familiar with the document structure of each XML source to formulate queries. Heuser $e t$ al. also present a new language (CXPath) based on XPath for querying XML sources at the conceptual level [8]. CXPath is used to write queries over a conceptual schema that abstracts the semantic content of several XML sources. However, they do not consider the situation of query translation from the XML sources to the global conceptual schema.

\subsection{Query rewriting}

Query rewriting or query translation is the key issue for both mediator-based integration systems and peer-to-peer systems. As an example of the first case, the Clio approach [18] mainly addresses schema mapping and data transformation between nested schemas and/or relational databases. It focuses on how to take advantage of schema semantics to generate the consistent translations from source to target by considering the constraints and structure of the target schema. The approach uses queries to express the mapping so as to transform the data into the target schema. The Piazza system [11] is a peer-to-peer system that aims to solve the problem of data interoperation between XML and RDF. The system achieves its interoperability in a low-level (syntactic) way, i.e., through the interoperability of XML and the XML serialization of RDF.

\section{Architecture}

In this section, we discuss the architecture based on the following two aspects: the integration of XML sources into the global ontology and the query processing within the integration system.

\subsection{Ontology integration and mapping table}

The ontology integration process contains two steps: schema transformation and ontology merging. In the first step, we use RDFS to model each XML source as a local RDF ontology to achieve a uniform representation basis for the ontology merging step. The key operation is the preservation of the nesting structure of the XML documents. In the second step, we merge all the local RDF schemas to generate the global ontology. In this process, additional domain-related knowledge (e.g., inheritance) may be introduced. During the merging process, a mapping table is produced to contain the mapping information between the global RDF ontology and local RDF ontologies. To simplify our discussion, we assume that the only semantics of the mappings is equivalence. We also do not consider the 


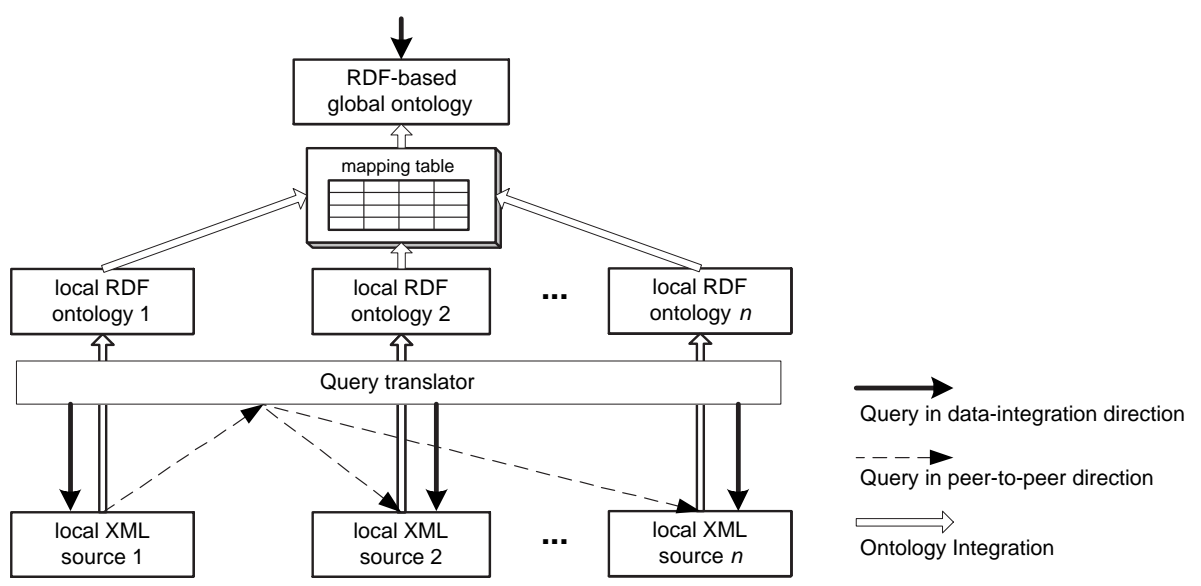

Figure 2. Architecture of the integration framework.

different degrees to which two concepts may be equivalent. For instance, we simply take book and article as equivalent concepts, although they might be semantically different in some cases. We discuss this process in more detail in Section 4.

It is worth mentioning that the global ontology in our system has two roles: (1) It provides the user access to the data with a uniform query interface to facilitate the formulation of a query on all the XML sources; (2) It serves as the mediation mechanism for accessing the distributed data through any of the XML sources.

\subsection{Query processing and reversibility}

As shown in Figure 2, the translation of queries in our system may occur in two directions:

Data integration The system translates an RDF query (directed to the global ontology) into multiple sub-queries (one for each XML source).

Peer-to-peer integration Query translation is performed in a similar way to query processing in peer-to-peer systems [19]. That is, the query posed by a user against any XML source is propagated to all the mediated distributed sources.

We use XQuery [4] to write queries over the XML sources and we use RDQL (RDF Data Query Language, based on SquishQL [12]) to write queries over the RDF global ontology.

The translation between structural queries in XQuery and conceptual queries in RDQL involves the problem of reversibility. In this subsection, we introduce the concept of reversibility that forms the basis for query translation across syntactically, schematically, and semantically heterogeneous data sources.

We start by looking at the problem for a single source. Intuitively, the query translation mechanism from a source query $Q$ to the target query $Q^{\prime}$ is said to be reversible when we can translate $Q^{\prime}$ to a query $Q^{\prime \prime}$, that produces an answer that is semantically equivalent to that produced by $Q$ on the data source. The left side of Figure 3 illustrates this case.

For distributed sources, the user poses a query $Q_{1}$ (called the source query) on the local XML source 1 , which is translated first to $Q_{g}$ on the global ontology. Then $Q_{g}$ is translated into multiple sub-queries $Q_{i}, 2 \leq i \leq n$ (called target queries), on the other $n-1$ distributed XML sources. A translation can be formalized as a function $f: Q_{g}=f\left(Q_{1}, M_{1}\right)$, where $Q_{g}$ is the query on the global ontology and $M_{1}$ is the set of mappings between the local $X M L$ source 1 and the global ontology. The translation $f$ is called reversible if and only if for each local XML source $i, Q_{i}=f^{-1}\left(Q_{g}, M_{i}\right)$ is equivalent to the source query $Q_{1}$ ( $M_{i}$ is the set of mappings between the global ontology and the local XML source i). Informally, two queries are equivalent if they return equivalent answer sets on semantically equivalent data sets. For example, the two XML documents of Figure 1 are semantically equivalent data sets. One just has to consider that $w_{i}$ is mapped to $a_{i}, 1 \leq i \leq 3$, and that $t_{i}$ is mapped to $b_{i}, 1 \leq i \leq 2$. Note that not only should this

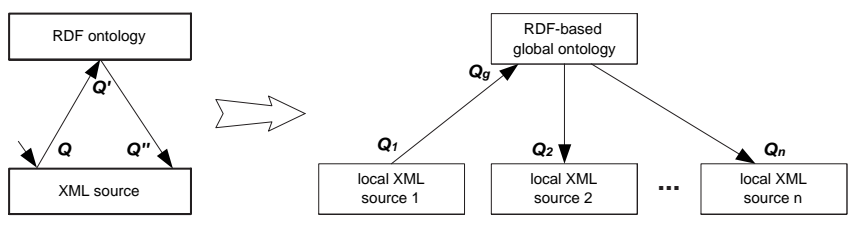

Figure 3. Query translation and reversibility. 
mapping be a bijection but also that other relationships need to be preserved. For example, in this case, the inverse of the nesting relationship is preserved. In Section 5, we use a concrete example (Example 5.3) that illustrates the concept of reversibility.

\section{Integrating Structure and Semantics: A Case Study}

To support the reversible query translation process between the structural query (XQuery) and the semantic query (RDQL), we choose to extend the vocabulary of RDF to make it capable of representing not only the semantics but also the structure of the data. In particular, we define a new RDF property $r d f x$ :contain ( $r d f x$ stands for the namespace where contain is defined) to enable RDF representation of the XML nesting structure. The data integration process consists of two sub-processes, schema transformation and ontology merging, which are discussed respectively in Section 4.1 and Section 4.2 through a case study. In the case study we integrate the two XML schemas $S_{1}$ and $S_{2}$ (see Figure 1). Figure 4 shows the RDF ontologies $\left(S_{1}^{\prime}\right.$ and $\left.S_{2}^{\prime}\right)$ that result respectively from the transformation of $S_{1}$ and $S_{2}$.

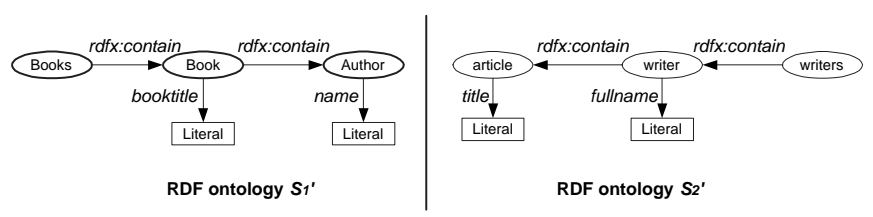

Figure 4. RDF ontologies transformed from the XML schemas in the case study.

\subsection{Local RDF ontology}

In the first sub-process, we transform the local XML schema into a local RDF ontology while preserving the XML document structure. By document structure, we mean the structural relationship of objects specified in datacentric documents [5] by a schema language (such as DTD, XML Schema, or RelaxNG). In this paper, we focus on the nesting structure (i.e., hierarchy). Other structural properties include order. A consequence of not including order in our framework is that we cannot consider a query $Q_{1}$ that involves the order of the subelements of an element. However, this kind of query is of little interest in a framework where we are mostly concerned with the semantics of the data.

In terms of XML Schema, elements and attributes are the two basic building blocks of XML documents. Ele- ments can be defined as simple types, which cannot have element content and cannot carry attributes, or complex types, which allow elements in their content and/or contain attributes. On the other hand, all attribute declarations must reference simple types since attributes cannot contain other elements or other attributes. From the perspective of XML Schema, these nesting relationships are defined in terms of datatypes (simple or complex type). A well-formed XML document contains the hierarchical structure of elements and attributes, which contains the following two aspects:

Element and attribute relationship Only complex-type elements can carry attributes and attributes can only be of simple types.

Element and sub-element relationship Likewise, only complex-type elements can allow elements as their children. But child elements can be either simple types or complex types.

Taking into account XML elements, attributes and their relationships, the transformation from XML to RDF can further include element-level transformation and structurelevel transformation.

Element-level transformation The element-level transformation defines the basic classes and properties of the local RDF ontology according to the transformation correspondences shown in Table 1, with the structural relationships between the elements not being considered for the time being. No new RDF metadata needs to be defined here because $r d f$ s:Class and $r d f s$ :Property are enough for the specifications of classes and properties. For instance, in the case study, for $S_{1}$ we define the classes: Books, Book, and Author while taking booktitle and name as properties of Book and Author, respectively (See Figure 4).

Table 1. Element-level transformation

\begin{tabular}{|l|l|}
\hline XML Schema concepts & RDF Schema concepts \\
\hline Attribute & Property \\
\hline Simple-type element & Property \\
\hline Complex-type element & Class \\
\hline
\end{tabular}

Structure-level transformation The structure-level transformation encodes the hierarchical structures of the XML schema into the local RDF ontology. The encoding involves two relationships: element-attribute relationship and element-subelement relationship. Following the element-level transformation, it is natural to encode the element-attribute relationship as a classto-literal relationship, and the element-subelement relationship as a class-to-class relationship in RDFS. We 
define a new RDFS predicate $r d f x$ :contain to represent class-to-class relationships. Specifically, we add a new property with its domain being one class (converted from the parent element), its range being the other class (converted from the subelement), and its name being rdfx:contain. As a result, we can see in Figure 4 that rdfx:contain enables the representation of the nesting relationship. For instance, by following the edges of $r d f x$ :contain from Books to Author in $S_{1}^{\prime}$, we in fact get the corresponding XPath /books/book/author in $S_{1}$. Table 2 lists the mapping information between the XML source $S_{1}$ and the local RDF ontology $S_{1}^{\prime}$.

Table 2. Mappings between $S_{1}$ and $S_{1}^{\prime}$

\begin{tabular}{|l|l|}
\hline XPath expressions in $S_{1}$ & RDF expressions in $S_{1}^{\prime}$ \\
\hline /books & Books \\
\hline /books/book & Book \\
\hline /books/book/@ booktitle & Book.booktitle \\
\hline /books/book/author & Author \\
\hline /books/book/author/@name & Author.name \\
\hline
\end{tabular}

\subsection{Global RDF ontology}

The process of ontology merging takes multiple local ontologies (encoded in RDFS) as the input and returns a merged ontology as the output [20]. Ontology merging and ontology alignment are widely pursued research topics. In this paper we do not intend to introduce a new technique for ontology merging. Instead, we utilize existing techniques to generate the integrated ontology from the local ontologies. In particular, we use an approach (such as PROMPT [16]) that provides the following functionalities:

- Merging of classes where multiple conceptually equivalent classes are combined into one class.

- Merging of properties where multiple conceptually equivalent properties of a class are combined into one property.

- Merging relationships between classes where conceptually equivalent relationships from one class $c_{1}$ to another class $c_{2}$ are combined into one relationship (i.e., an RDF property taking $c_{1}$ as its domain and $c_{2}$ as its range).

- Copying a class and/or its properties if the same or equivalent class/property does not exist in the target ontology.

- Generalizing related classes into a more general superclass. The superclass can be obtained by searching an existing knowledge domain (e.g., the DAML Ontology Library) or reasoning over a thesaurus.

Figure 5 gives the ontology that results from merging two local RDF ontologies (see Figure 4) in the case study. The grayed classes and properties are merged classes and properties from the original ontologies. For instance, the class Book is merged from Book in $S_{1}^{\prime}$ and Article in $S_{2}^{\prime}$, whereas the property title is merged from booktitle in $S_{1}^{\prime}$ and title in $S_{2}^{\prime}$. The classes Book and Author are also respectively extended with the superclasses Publication and Person.

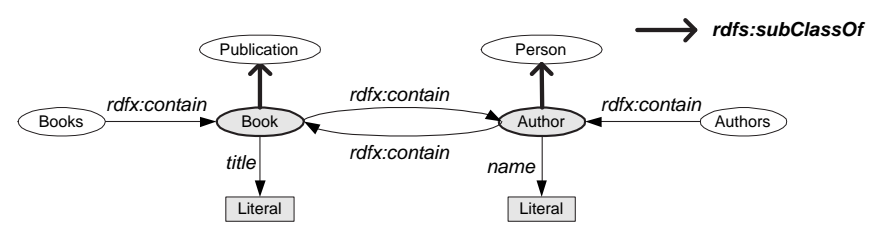

Figure 5. The global ontology merged from local RDF ontologies.

Apart from the global ontology, the ontology merging sub-process also yields another output: the mapping table that contains the mapping information between the local RDF ontologies and the global RDF ontology. In general, if a class, property, or relationship between classes $p$ in the global ontology is the result of merging $p_{i}$ and $p_{j}$ from different local ontologies, then a mapping in the form of $\left(p, p_{i}, p_{j}\right)$ is generated. If a class or property $p$ in the global ontology is only copied from $p_{i}$ in a local ontology, then a mapping $\left(p, p_{i}\right)$ is produced. For instance, for the class Book.title (in the global ontology), which is merged from Book.booktitle in $S_{1}^{\prime}$ and Article.title in $S_{2}^{\prime}$, we generate an entry in the mapping table: (Book.title, Book.booktitle, Article.title). Table 3 lists all the mappings in our case study.

Table 3. Mapping table between the global ontology and local RDF ontologies

\begin{tabular}{|l|l|l|}
\hline $\begin{array}{l}\text { RDF expressions in } \\
\text { the global ontology }\end{array}$ & $\begin{array}{l}\text { RDF expre- } \\
\text { ssions in } S_{1}^{\prime}\end{array}$ & $\begin{array}{l}\text { RDF expre- } \\
\text { ssions in } S_{2}^{\prime}\end{array}$ \\
\hline Books & Books & - \\
\hline Book & Book & Article \\
\hline Book.title & Book.booktitle & Article.title \\
\hline Authors & - & Writers \\
\hline Author & Author & Writer \\
\hline Author.name & Author.name & Writer.fullname \\
\hline
\end{tabular}




\section{Query Processing}

The system can process queries in two directions: the data-integration direction, the query on the global ontology is rewritten into subqueries over multiple sources, and the peer-to-peer direction, the query on some XML source is propagated to the XML sources connected through the global ontology. In this section, we describe the query rewriting algorithm by using concrete examples.

\subsection{Assumptions for the query rewriting algo- rithm}

To represent queries, RDQL uses an SQL-like syntax, in which the SELECT clause identifies the variables to be returned to the application. The From clause specifies the RDF model using an URI. The Where clause specifies the graph pattern as a list of triple patterns. The And clause specifies the Boolean expressions. Finally, the Using clause provides a way to shorten the length of the URIs. Whereas XQuery is a functional programming language which has an FLWR (i.e., For, Let, Where, Return) syntax. For simplification, we make the following assumptions for the query rewriting algorithm.

- We assume that the XML sources to be integrated are related to the same knowledge domain, and data instances are well populated in these sources so that we do not necessarily consider handling null values from the XML sources.

- We also assume that the XML query posed by the user is formulated only in the form of FLWR expressions [4]. However, we are not going to discuss translation of nesting XML queries in the rewriting algorithm, although nesting FLWR expressions are allowed in XQuery.

- We finally assume that all the concepts in every local ontologies are mapped to the concepts in the global ontology during the ontology integration process. That is, the mappings are total, one-to-one mappings from the local RDF ontologies to the global ontology. It is possible that some concept, say $c_{i}$, in the global ontology gets mapped to a local ontology but not to another local ontology. This leads to the problem of null values when a query involves $c_{i}$.

\subsection{From RDQL to XQuery}

The global ontology connects all the individual local RDF ontologies that represent local XML sources and provides a uniform query interface for the user. Thus, the user can retrieve data from all the sources in the system by simply submitting a single RDF query on the global ontology. In this subsection, we discuss the translation from an RDQL query on the global ontology to XQuery queries over the XML sources. In the following discussion, we use $M$ to denote the mapping table (between the global ontology and the local RDF ontologies), $Q_{g}$ for the input RDQL query (on the global ontology), $Q_{r}$ for the intermediate query (on the local RDF ontology), and $Q_{x}$ for the output XQuery query (on the XML source). The process of query rewriting from RDQL to XQuery proceeds as follows:

Step 1 Identify the RDF path expression corresponding to each variable that is used in $Q_{g}$, put these RDF path expressions into a set $P$, and group them into different sets based on their different roles: (1) Put the RDF expression that appears in the Select clause into a set $P_{s}$. (2) Put the RDF expression, which appears in the Where clause and is constrained with a Literal constant or a URI, into a set $P_{w}$. (3) Also put the RDF expression that occurs in the And clause into $P_{w}$.

Step 2 For each participating local RDF ontology $R_{i}$, based on the correspondences in the mapping table $M$, replace the RDF paths in $P$ with the RDF paths mapped in $R_{i}$, while updating $P_{s}$ and $P_{w}$ in the same way. Rewrite $Q_{g}$ into $Q_{r}$ by using the following method: (1) For the Where clause, traverse $R_{i}$ to find an acyclic sub-graph that covers all the path expressions in $P$, and put all the edges (properties) into a set $E$. For each $e_{i} \in E$ (with the subject $s_{i}$ and the object $\left.o_{i}\right)$, add a triple in the form of $\left(? s_{i}, e_{i}, ? o_{i}\right)$ into the Where clause. (2) For the Select and And clauses, simply replace the RDF paths in them with their corresponding paths in $R_{i}$, then bind each path with a variable.

Step 3 Find the XPath expression corresponding to every element $p_{i}$ in both $P_{s}$ and $P_{w}$, by utilizing the mapping information between the local XML source and its local RDF ontology $R_{i}$. For the result, we use $P_{s}^{\prime}$ to denote the set of XPaths corresponding to $P_{s}$, and $P_{w}^{\prime}$ for $P_{w}$.

Step 4 Construct the target query $Q_{x}$ for each XML source according to the following rules:

- The Let Clause: Output let $\$<$ root label $>$ $:=\operatorname{doc}("<X M L$ source name $>")$.

- The For Clause: For each XPath $p_{i}$ in both $P_{w}^{\prime}$ and $P_{s}^{\prime}$, output a For clause in the form of for $<$ node label $>$ in $\left\langle p_{i}>\right.$.

- The Where Clause: For each $p_{i}^{\prime}$ in $P_{w}^{\prime}$, construct a query condition according to the constraints in 
$Q_{r}$, and take the conjunction of these conditions as the Where clause.

- The Return Clause: Take each $p_{i}^{\prime}$ in the set $P_{s}^{\prime}$ as an element in the Return clause.

Step 5 Abbreviate each absolute XPath $p_{i}$ referred in $Q_{x}$ into a relative XPath by replacing some part of $p_{i}$ with $v$, if this part was bound to $v$.

Example 5.1 Suppose we submit in our case study a query $Q_{g}$ on the global ontology: "List all the book titles," whose RDQL-syntax code is shown below. We assume that the global ontology is defined in the namespace: http://examples.org/go\# and the local ontology $R_{i}$ is defined in the namespace: http://examples.org/lo-i\# (we will be making the same assumptions in all the examples of this section). In this example, we illustrate the algorithm by translating $Q_{g}$ into a query on books.xml (see Figure 1).

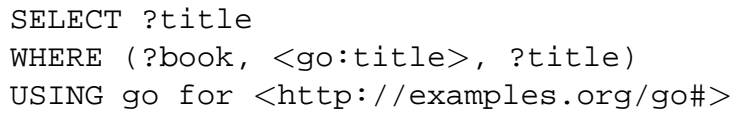

After Step 1, we get $P=\{$ Book, Book.title $\}, P_{s}$ $=\{$ Book.title $\}$, and $P_{w}$ is empty. By looking into the mapping information in Table 3, we update $P=\{$ Book, Book.booktitle $\}$ and $P_{s}=\{$ Book.booktitle $\}$, and rewrite $Q_{g}$ into $Q_{r}$ as shown below (after Step 2).

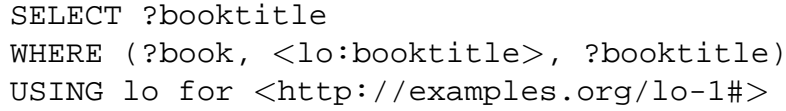

In Step 3, we find that /books/book/ abooktitle in books.xml is the corresponding XPath of Book.booktitle in the local ontology $R_{1}$. Thus, $P_{s}^{\prime}=\{/$ books/book/abooktitle $\}$ and $P_{w}^{\prime}$ is still empty. By following the XQuery construction instructions in Step 4 and XPath abbreviation method in Step 5, we output the target query $Q_{x}$ as:

let \$books := doc ("books.xml")

for \$booktitle in \$books/book/abooktitle

return \$booktitle

Example 5.2 Suppose a query $Q_{g}$ "List all the books written by $a_{2}$ " is posed by the user over the global ontology. We are supposed to translate it into a query $Q_{x}$ against writers.xml.

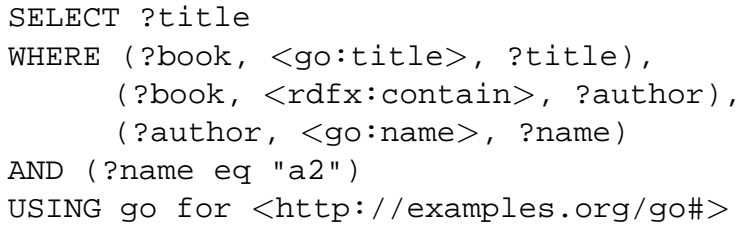

After Step 1, we get $P=\{$ Book, Book.title, Author, Author.name $\}, P_{s}=\{$ Book.title $\}$, and $P_{w}=$ \{Author.name . In Step 2, by looking into the mapping table $M$, we update $P$ $=\quad$ Article, Article.title, Writer, Writer.fullname,$\quad P_{s}=$ AAticle.title , $P_{w}=\{$ Writer.fullname $\}$, and rewrite $Q_{g}$ into $Q_{r}$ as shown below.

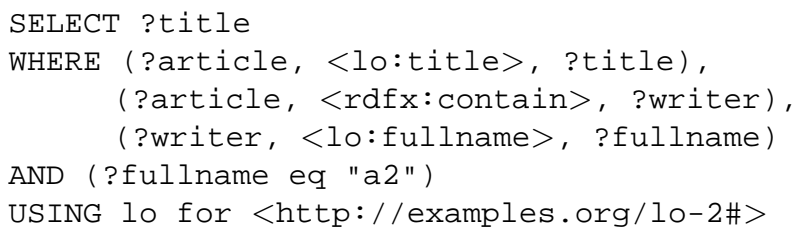

In Step 3, we get $P_{s}^{\prime}=$ \{/writers/ writer/@fullname $\}$ and $P_{w}^{\prime}=\{/$ writers/writer /article/@title\}. By following the XQuery construction instructions in Step 4 and XPath abbreviation method in Step 5, the resulting target query $Q_{x}$ as follows:

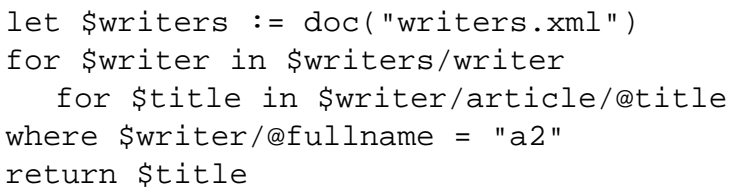

\subsection{Peer-to-peer integration}

The query rewriting in the peer-to-peer integration direction contains three phases: (1) The input XQuery query $Q_{x}$ posed by the user on an XML source is translated into an RDQL query $Q_{r}$ over its corresponding local RDF ontology. (2) $Q_{r}$ is translated into an equivalent RDQL query $Q_{r}^{\prime}$ over each peer local RDF ontology by using the mapping information in Table 2. (3) $Q_{r}^{\prime}$ is rewritten into the query $Q_{x}^{\prime}$ over the XML source. It is obvious that the last two phases proceed in the same way as the query rewriting in the data-integration direction. The first phase consists of the following steps:

Step 1' Extend the relative XPaths referred to in the Where clause and in the Return clause of the original XML query respectively into a set of absolute XPaths, denoted by $P_{1}$ for the Where clause and by $P_{2}$ for the Return clause.

Step 2' Based on the mapping information between the local XML source and its RDF ontology $R_{i}$, we can get the RDF path expressions corresponding to the XPaths in both $P_{1}$ and $P_{2}$, using $P_{1}^{\prime}$ and $P_{2}^{\prime}$ respectively to denote the resulting sets of RDF paths for $P_{1}$ and $P_{2}$.

Step 3' Construct the query $Q_{r}$ on $R_{i}$ according to the following rules. 


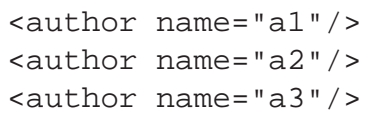

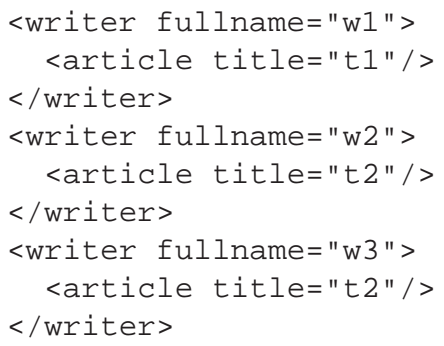

Figure 6. An example of equivalent answer sets

- SELECT clause: Bind each RDF path $p_{i}$ in $P_{2}^{\prime}$ to a variable $v_{i}$, whose name is the label of $p_{i}$ in the RDF ontology. Add each $v_{i}$ into the SELECT clause as an item and output: SELECT $\quad ?<v_{1}>$, $\ldots,<v_{i}>, \ldots,<v_{n}>$.

- WHERE clause: Traverse $R_{i}$ to find an acyclic sub-graph that covers all the RDF expressions in both $P_{1}^{\prime}$ and $P_{2}^{\prime}$, and put all the edges (properties) into the set $E$. For each $e_{i}$ (with $s_{i}$ as its subject and $o_{i}$ as its object) in $E$, add a triple in the form of $\left(? s_{i}, e_{i}, ? o_{i}\right)$ into the WHERE clause.

- AND clause: For each $p_{i}$ in $P_{1}^{\prime}$, construct a query condition according to the original constraints in $Q_{x}$. Take the conjunction of these conditions as the AND clause.

- FROM and USING clauses: These clauses are related to URIs and therefore are not further discussed.

Example 5.3 Suppose the user posed the original query $Q_{x}$ : "List all the authors" on the XML source writers.xml. Our algorithm will rewrite it into a query on the other XML source books. xml. The XQuery for the original query can be written as follows.

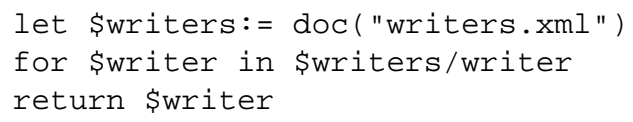

After Step 1', we obtain an empty set $P_{1}$ and $P_{2}=$ \{/writers/writers\}. After Step 2', $P_{1}^{\prime}$ is an empty set and we have $P_{2}^{\prime}=\{$ Writer $\}$. Following the instructions in Step 3', we can construct the RDQL query $Q_{r}$ that only refers to the class Writer in the local ontology $S_{2}^{\prime}$ (see Figure 4). By simply applying the rewriting algorithm from RDQL to XQuery on $Q_{r}$, we finally get query $Q_{x}^{\prime}$ (on books.xml):

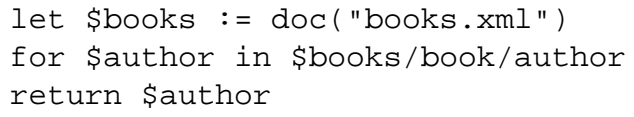

As discussed in Section 3.2, the query translation from XQuery through RDQL to XQuery involves the concept of reversibility. We have shown that the translation reversibility depends on the equivalence between the source query $\left(Q_{x}\right.$ in Example 5.3) and the target query $\left(Q_{x}^{\prime}\right.$ in Example 5.3), which depends on the equivalence of the answer sets produced by $Q_{x}$ and by $Q_{x}^{\prime}$. In Example 5.3, the answer sets to $Q_{x}$ and $Q_{x}^{\prime}$ are shown in Figure 6 (respectively on the left and on the right), where we can see that the two answer sets are structurally different but semantically equivalent.

\section{Conclusions and Future Work}

XML and its schema languages do not express semantics but rather the document structure, such as the information about nesting. Therefore, semantically-equivalent documents often present different document structures when they originate from different applications. In this paper, we provide an ontology-based framework that aims to make XML documents interoperate at the semantic level while retaining their nesting structure. The framework consists of two key aspects: the mapping process and query processing.

In the mapping process, an RDF-based global ontology is generated by merging the RDF ontologies that are generated from each of the XML documents. At the same time, the mappings between the global ontology and local XML schemas are established, which is done manually. We extend RDFS by defining additional metadata that can encode the nesting structure of an XML document. For query processing, we propose two query rewriting algorithms: one algorithm translates an RDF query (posed on the global ontology) to an XML query; the other algorithm translates an XML query (posed on one of the individual XML data sources) to another XML query (posed on a different XML data source). The last query processing mechanism is therefore bidirectional and corresponds to a P2P mode of operation. We introduce the concept of reversibility to guarantee the correctness of the bidirectional query processing.

Future work will include: (1) Proving the correctness of our query translation algorithms. (2) Proposing an approach 
for the unification of the results (as expressed in XML) with different structures, which are returned from different sources.

\section{References}

[1] B. Amann, C. Beeri, I. Fundulaki, and M. Scholl. Ontology-Based Integration of XML Web Resources. In Proceedings of the 1st International Semantic Web Conference (ISWC 2002), pages 117-131, 2002.

[2] B. Amann, I. Fundulaki, M. Scholl, C. Beeri, and A. Vercoustre. Mapping XML Fragments to Community Web Ontologies. In Proceedings of the 4th International Workshop on the Web and Databases (WebDB 2001), pages 97-102, 2001.

[3] Y. Bishr. Overcoming the semantic and other barriers to GIS interoperability. International Journal of Geographical Information Science, 12(4):229-314, 1998.

[4] S. Boag, D. Chamberlin, M. F. Fernández, J. R. D. Florescu, and J. Siméon. XQuery 1.0: An XML Query Language. http://www.w3.org/TR/xquery, W3C Working Draft, August 2003.

[5] R. Bourret. XML and Databases. http://www.rpbourret.com/xml/XMLAndDatabases.htm.

[6] D. Brickley and R. Guha. RDF Vocabulary Description Language 1.0: RDF Schema. http://www.w3.org/TR/rdf-schema, W3C Working Draft, January 2003.

[7] A. Calì, D. Calvanese, G. D. Giacomo, and M. Lenzerini. On the Expressive Power of Data Integration Systems. In 21 st Intl. Conference on Conceptual Modeling (ER), pages 338-350, 2002.

[8] S. D. Camillo, C. A. Heuser, and R. S. Mello. Querying Heterogeneous XML Sources through a Conceptual Schema. In Proceedings of the 22nd International Conference on Conceptual Modeling (ER2003), pages 186-199, 2003.

[9] Y. Chen and P. Revesz. CXQuery: A Novel XML Query Language. In Proceedings of International Conference on Advances in Infrastructure for Electronic Business, Science, and Medicine on the Internet (SSGRR 2002w), 2002.

[10] I. F. Cruz and H. Xiao. Using a Layered Approach for Interoperability on the Semantic Web. In Fourth International Conference on Web Information Systems Engineering (WISE'03), pages 221-232, Roma, Italy, December 2003.
[11] A. Y. Halevy, Z. G. Ives, P. Mork, and I. Tatarinov. Piazza: Data Management Infrastructure for Semantic Web Applications. In Proceedings of the 12th International World Wide Web Conference (WWW2003), pages 556-567, 2003.

[12] HP Labs. RDQL - RDF Data Query Language. http://www.hpl.hp.com/semweb/rdql.htm.

[13] M. C. A. Klein. Interpreting XML Documents via an RDF Schema Ontology. In Proceedings of the 13th International Workshop on Database and Expert Systems Applications (DEXA 2002), pages 889-894, 2002.

[14] L. V. Lakshmanan and F. Sadri. Interoperability on XML Data. In Proceedings of the 2nd International Semantic Web Conference (ICSW'03), 2003.

[15] F. Manola and E. Miller. RDF Primer. http://www.w3.org/TR/rdf-primer, W3C Working Draft, January 2003.

[16] F. Noy and M. A. Musen. PROMPT: Algorithm and Tool for Automated Ontology Merging and Alignment. In Proceedings of the Seventeenth National Conference on Artificial Intelligence and Twelfth Conference on Innovative Applications of Artificial Intelligence (AAAI/IAAI 2000), pages 450-455, 2000.

[17] P. F. Patel-Schneider and J. Siméon. The Yin/Yang web: XML syntax and RDF semantics. In Proceedings of the 11th International World Wide Web Conference (WWW2002), pages 443-453, 2002.

[18] L. Popa, Y. Velegrakis, R. J. Miller, M. A. Hernandez, and R. Fagin. Translating web data. In Proceedings of $V L D B$, pages 598-609, 2002.

[19] O. D. Sahin, A. Gupta, D. Agrawal, and A. E. Abbadi. Query Processing Over Peer-To-Peer Data Sharing Systems. Technical Report CSD-2002-28, University of California at Santa Barbara, 2002.

[20] G. Stumme and A. Maedche. Ontology Merging for Federated Ontologies on the Semantic Web. In Proceedings of the International Workshop for Foundations of Models for Information Integration (FMII2001), pages 413-418, 2001. 